\title{
Image cytometry as an alternative to flow cytometry for the transplant histocompatibility crossmatch assay
}

\author{
Daniel S. Ramon $^{1,2}$ (D) | Thomas Franks ${ }^{2}$ | Andrés Jaramillo ${ }^{1}$ | Benjamin D. Paradis ${ }^{3}$ | \\ Leo Li-Ying Chan $^{3}$
}

${ }^{1}$ Department of Laboratory Medicine and Pathology, Mayo Clinic, Phoenix, Arizona

${ }^{2}$ Department of Pathology, University of Michigan School of Medicine, Ann Arbor, Michigan

${ }^{3}$ Department of Technology R\&D, Nexcelom Bioscience LLC, Lawrence, Massachusetts

\section{Correspondence}

Daniel S. Ramon, PhD, Department of Laboratory Medicine and Pathology, Mayo Clinic, 5777

E. Mayo Blvd., Phoenix, AZ 85054.

Email: ramon.daniel@mayo.edu
The lymphocyte crossmatch is currently the only cell-based compatibility assay performed by histocompatibility laboratories for transplant purposes. While in many transplant programs the complement-dependent cytotoxicity crossmatch (CDCXM) remains in use, when available, the flow cytometry crossmatch (FCXM) is the method of choice because of its superior sensitivity and specificity. Unfortunately, the maintenance and cost of a flow cytometer is a considerable limitation for small histocompatibility laboratories. Therefore, in this study, we evaluated the use of the Cellometer Vision CBA image cytometer (Nexcelom Bioscience LLC, Lawrence, Massachusetts) as an alternative instrument to perform the crossmatch assay. The 3-color FCXM protocol was modified into two separate 2-color panel image cytometry crossmatches (IXMs), one for T cells and one for B cells. After initial serum and cell incubation, a cocktail consisting of PE/Cy5-conjugated anti-human CD3 or CD19 and PE-conjugated anti-human IgG $\mathrm{F}\left(\mathrm{ab}^{\prime}\right)_{2}$ was added to the $\mathrm{T}$ cell and $\mathrm{B}$ cell panels, respectively. The final cell preparation was added to a separate counting chamber. Images were captured using the Cellometer Vision CBA, an image cytometer designed for cell counting, size analysis and fluorescence intensity measurement. Thirty-nine IXMs were performed and compared with the FCXM. We obtained a concordance sensitivity of $94.1 \%$ and $100 \%$ and specificity of $100 \%$ and $88.9 \%$ for T cells and B cells, respectively. The linearity of the system was verified using dilutions of a sample containing known donor-specific anti-HLA antibodies (DSA) against the target cells. This feasibility study demonstrates that the FCXM test could be easily adapted to the Cellometer Vision CBA image cytometer without compromising specificity and sensitivity. The low instrumentation cost, minimal maintenance, and simple operation allow for efficient implementation or transition from the FCXM to the IXM method.

\section{KEYWORDS}

crossmatch, donor-specific anti-HLA antibodies, flow cytometry, histocompatibility, human leukocyte antigens, image cytometry, transplantation

\section{1 | INTRODUCTION}

Organ transplantation is a well-established treatment for progressive and irreversible organ failure. Two large immunologic barriers must be overcome before most solid organ transplant procedures. The first is the compatibility of $\mathrm{ABO}$ blood groups, and the second is the presence of preformed anti-HLA antibodies in the serum of the organ recipient. The crossmatch reaction has been an essential tool to facilitate organ distribution, donor selection, and post-transplant risk assessment. With the original complement-dependent cytotoxicity crossmatch $(\mathrm{CDCXM})$ reaction, a positive result 
was associated with hyperacute or accelerated rejection, produced by massive complement cascade activation due to the high concentration of donor-specific anti-HLA antibodies (DSA) demonstrated by the pivotal work of Patel and Terasaki. ${ }^{1}$

The low sensitivity to detect DSA was the main limitation of the CDCXM. This assay was later modified to improve its sensitivity and specificity with the addition of washing steps or incubation with anti-human globulin $(\mathrm{AHG})^{2,3}$ without substantial changes in the performance characteristics of the assay. Despite these limitations, the CDCXM remains in use in many laboratories around the world and it is a requirement for transplantation in many programs.

A considerable improvement in sensitivity and specificity was achieved with the introduction of the flow cytometry crossmatch (FCXM). ${ }^{4}$ This and other studies showed that the FCXM was capable of detecting low DSA levels not previously detected by the CDCXM. ${ }^{5-8}$ Most notably, renal transplant recipients exhibiting negative CDCXM or AHG-augmented CDCXM, but positive FCXM, were more likely to experience early accelerated rejection and graft loss. ${ }^{9-12}$ Hence, for the assessment of alloantibody reactivity, in those laboratories with access to a flow cytometer, the FCXM replaced the CDCXM as the pre-transplant crossmatch assay.

Simultaneously, new solid-phase methods became available to the histocompatibility laboratory for the detection and characterization of anti-HLA antibodies, allowing for a crossmatch prediction algorithm known as the virtual crossmatch (VXM) ${ }^{13}$ However, despite the reliability of the VXM, many transplant centers, combined the information from solid-phase methods and the FCXM for the final transplant decision and risk assessment.

Without a doubt, the flow cytometer is an excellent instrument to perform the FCXM assay. Among the benefits that can be highlighted are the high sensitivity and specificity, the simultaneous detection of multiple analytes, and the more than 30 years of experience of clinical application. This instrument is constructed with multiple lasers to excite a large number of fluorescent labels and a sophisticated optical system to collect and filter the emission fluorescent light, as well as to analyze the size and internal complexity of a large number of particles. Furthermore, the instrument is designed with an intricate pneumatic and fluidic system; thus, the acquisition cost of this instrument is relatively high. In addition, the complex setting and operation of the flow cytometer, as well as laborious maintenance, can make training difficult and requires a considerable amount of time.

Substantial technological advances in the acquisition of digital images, microfluidics and compact light-emitting diodes (LEDs) as a source of fluorochrome excitation light have allowed the development of a simple and compact type of instrument capable of reproducing some of the functions performed using the flow cytometer. The replacements of expensive and bulky lasers and the complex fluidic systems by compact and affordable LED bulbs and microfluidic chambers have given rise to small footprint benchtop instruments with lower acquisition costs and virtually no maintenance cost. With this currently available technology, the crucial concern is whether the flow cytometer remains the most adequate instrument for a relatively simple detection like the crossmatch assay.

In this study, we explored the possibility of employing an image cytometer instrument, the Cellometer Vision CBA (Nexcelom Bioscience LLC), as an alternative instrument to perform the crossmatch assay. We adapted our FCXM protocol to determine whether the crossmatch assay could be analyzed and interpreted by the image cytometer. As a result, we validated the performance of the image cytometry crossmatch (IXM) protocol by comparing the results with the FCXM results as the reference methodology.

\section{2 | MATERIALS AND METHODS}

\section{1 | Three-color FCXM}

Our laboratory used a 3-color FCXM procedure described previously. ${ }^{6}$ Briefly, $5 \times 10^{5}$ lymphocytes were suspended in wash buffer ( $5 \%$ fetal bovine serum and $0.1 \%$ sodium azide in PBS) and then centrifuged at $3000 \mathrm{~g}$ in a $12 \times 75$ polystyrene tube. Forty microliters of the appropriate sample (NHS [normal human serum], PHS [positive human serum], and test sera), previously centrifuged at $14000 \mathrm{~g}$, was added to the cells and incubated at room temperature for 30 minutes. Subsequently, the cells were washed 3 times with wash buffer. After decanting the wash buffer, $20 \mu \mathrm{L}$ of FITC-conjugated $\mathrm{F}\left(\mathrm{ab}^{\prime}\right)_{2}$ goat anti-human $\operatorname{IgG}$ (Fc-specific) polyclonal antibody (Jackson ImmunoResearch Laboratories, Inc, West Grove, Pennsylvania; Cat. No. 109-096-098) and $30 \mu \mathrm{L}$ of a mix of PE/Cy5-conjugated mouse anti-human CD3 and PEconjugated mouse anti-human CD19 monoclonal antibodies (mAb) (Beckman Coulter, Inc., Brea, California; Cat. No. IM2635/IM1285) were added to each tube and incubated at room temperature for 20 minutes in the dark. Next, the cells were washed twice with wash buffer and resuspended. The FCXM was analyzed in an FC500 Beckman Coulter flow cytometer (Beckman Coulter, Inc.). Forward vs side scatter and CD3 vs CD19 dot plots were used to analyze $\mathrm{T}$ cell and B cell populations individually. From a histogram with the cell count and the FITC signal intensity, the median channel (MC) for each sample was determined. Fluorescence median channel shift (MCS) was calculated by subtracting the NHS fluorescence MC value from the PHS and test sera fluorescence MC values. 
The MCS values were compared to the previously calculated cut-off value for positive or negative assignment. ${ }^{7,14}$

\subsection{Image cytometry instrumentation and disposable counting chamber}

The Cellometer Vision CBA image cytometer (Nexcelom Bioscience, LLC) has been described previously. ${ }^{15-17}$ The system utilized bright-field (BR) and dual-fluorescent (FL1 and FL2) imaging modes to quantitatively analyze and measure the fluorescence intensities of target cells. BR imaging used a white LED, and fluorescent imaging used a combination of monochromatic LEDs (527 and $624 \mathrm{~nm}$ ) as excitation light sources. The monochromatic LEDs were integrated into specific fluorescence optics modules (excitation/emission), VB-595-502 (525 $\pm 32 / 605 \pm 22 \mathrm{~nm})$ for PE and VB-695-502 $(525 \pm 32 / 695 \pm 30 \mathrm{~nm})$ for PE/ Cy5 detection. The system uses a magnification objective of $5 \times$ and the optical detection limit has been previously described. ${ }^{15}$ Typically, the fluorescently labeled cell sample is pipetted into a Nexcelom disposable counting chamber, which holds precisely $20 \mu \mathrm{L}$ of volume. The counting slide is held in position by a stage, which automatically moves to four locations on the chamber for cellular analysis by the Cellometer Vision CBA instrument's software. The software analyzes three image channels (BR, FL1, and FL2) and generates a fluorescent dataset that is automatically exported to FCS Express 4 image cytometry software (De Novo Software, Los Angeles, California).

\subsection{Two-color image cytometry crossmatch}

The 3-color FCXM panel was adapted to two separate 2-color T cell and B cell panels, respectively. For the T cell panel we used PE/Cy5-conjugated mouse anti-human CD3 mAb (Immunotech, Beckman Coulter; Cat. No. IM2635U) and PE-conjugated $\mathrm{F}(\mathrm{ab})_{2}$ goat anti-human $\mathrm{IgG}$ (Fc-specific) polyclonal antibody (Jackson ImmunoResearch Laboratories, Inc.; Cat. No. 109-116-098). The only difference on the $\mathrm{B}$ cell panel was the replacement of the $\mathrm{PE} / \mathrm{Cy} 5$ -conjugated mouse anti-human $\mathrm{CD} 3 \mathrm{mAb}$ by the $\mathrm{PE} / \mathrm{Cy} 5$ -conjugated mouse anti-human CD19 mAb (Immunotech, Beckman Coulter; Cat. No. IM2643U). Besides the changes in the mAbs, the rest of the crossmatch reactions remained consistent with the FCXM protocol. Parameters such as cell number, incubation time, and concentration and volume of reagents were maintained. After the suspension of the cells with wash buffer, $20 \mu \mathrm{L}$ of each reaction was dispensed into each side of the Nexcelom disposable counting chamber (Figure 1).

Four sets of BR, FL1, and FL2 fluorescent images were collected from each chamber containing the NHS, PHS, and test serum crossmatch reactions. The images were segmented and exported as .NXDAT files to be analyzed by the FCS Express 4 image cytometry software (Figure 2).

\section{4 | Parallel study}

\subsection{1 | Cell preparation}

The contents of 3 to 4 ACD blood collection tubes (20-25 mL) were transferred into a 50-mL conical tube. Forty $\mu \mathrm{L}$ of RosetteSep (Stemcell Technologies Inc., Vancouver, BC, Canada) were added, and the sample was vortexed gently and then incubated for 10 minutes at room temperature. The sample was overlaid onto the FicollHypaque chamber in the SepMate tube (Stemcell Technologies, Inc.). After centrifugation at $1200 \mathrm{~g}$ for 20 minutes, the upper supernatant was transferred into a $50-\mathrm{mL}$ conical tube. Next, the tubes were centrifuged at $1200 \mathrm{~g}$ for 5 minutes, where the cell pellet was washed twice with RPMI-1640 medium and resuspended in $2 \mathrm{~mL}$ of wash buffer. Subsequently, the cells were counted with the volume adjusted to a concentration of $5 \times 10^{6}$ cells $/ \mathrm{mL}$. Next, the tubes were centrifuged at $1200 \mathrm{~g}$ for 5 minutes. After removal of the supernatant, $1 \mathrm{~mL}$ of pronase solution (2.8 units protease $/ \mathrm{mL}$ ) was added and the cells were incubated at $37^{\circ} \mathrm{C}$ for 10 minutes. Finally, the cells were washed with RPMI1640 medium supplemented with $10 \%$ fetal bovine serum and washed twice with wash buffer with the concentration adjusted to $2.5 \times 10^{6}$ cells $/ \mathrm{mL}$. All samples were typed by SSO method (LABType, One Lambda, Inc., Canoga Park, California) for HLA-A, B, C, DRB1, DRB3/4/5, DQA1, and DQB1, and for HLA-DPA1 and DPB1 in cases with antibodies against HLA-DP molecules.

\section{5 | Serum samples}

Thirty-nine patient sera were tested in this study. The detection and characterization of anti-HLA antibodies was performed utilizing a Luminex single antigen bead (SAB) array (LABScreen, One Lambda, Inc.) following the manufacturer instructions. The product of this reaction was analyzed by the Luminex LABScan 200 flow analyzer (Luminex Corporation, Austin, Texas), where the raw data was collected and analyzed by Fusion software (One Lambda, Inc.). The strength of anti-HLA antibodies was expressed as mean fluorescence intensity (MFI) units.

Commercially available pooled serum from healthy $\mathrm{AB}$ donors (Gemini Bio-Products, West Sacramento, California; Cat. No. NC9833877) was used as negative control (NHS). The positive control (PHS) was obtained by pooling sera from multiple highly sensitized patients (PRA $\geq 80 \%$ ) with confirmed antibodies against both HLA class I and class II antigens by Luminex SAB array.

\subsection{Statistical analysis}

Differences in IXM and FCXM reactivity were analyzed by means of linear regression and Fisher exact tests using GraphPad Prism version 6.07 for Windows (GraphPad Software, Inc., La Jolla, California) with the alpha set at $P<0.01$. 

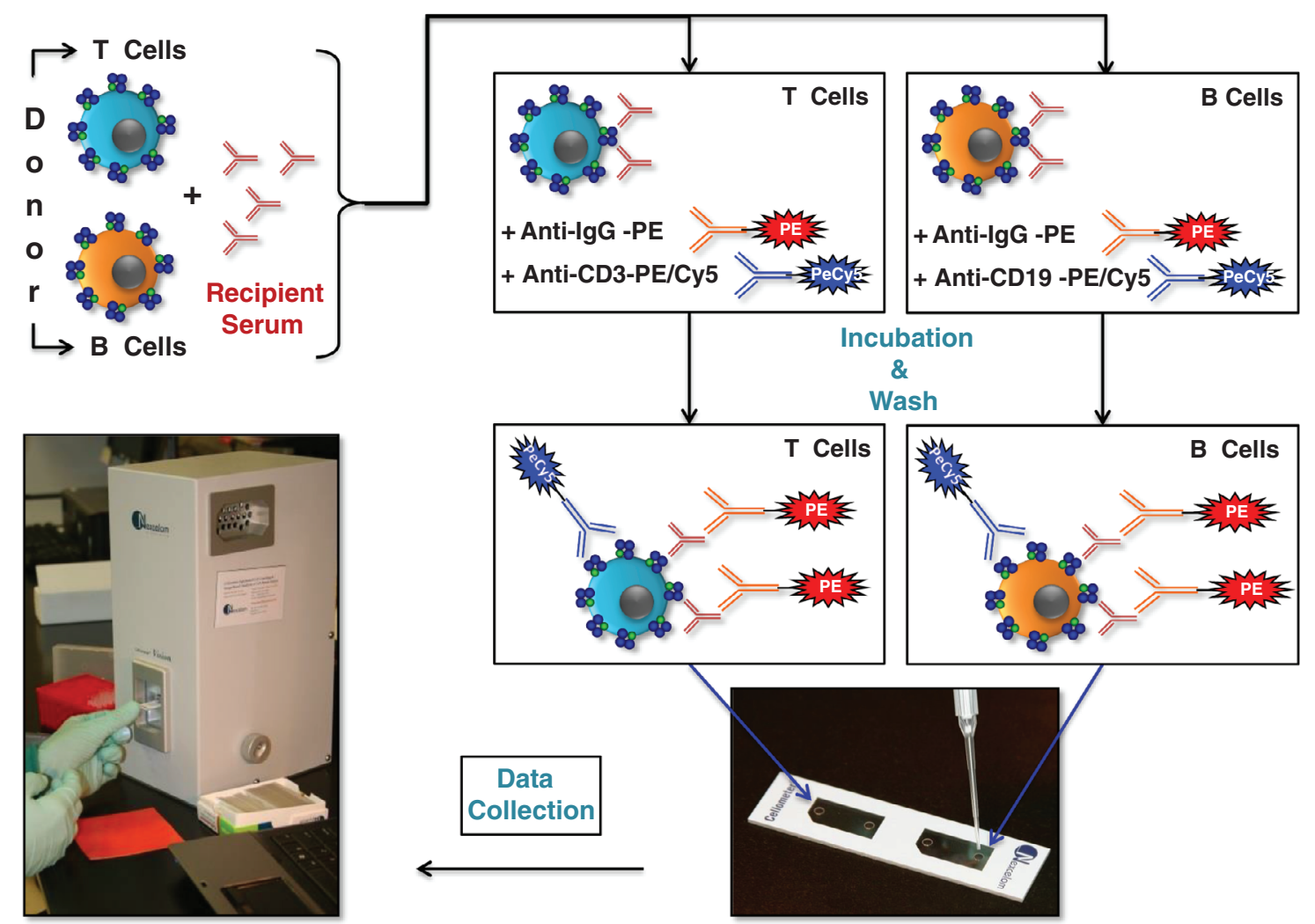

FIGURE 1 Two-color image cytometry crossmatch protocol. The 2-color T cell and B cell image cytometry crossmatch (IXM) protocol was adapted from the 3-color flow cytometry crossmatch (FCXM) protocol. Besides the reduction in fluorochromes, the rest of the reactions remained consistent with the FCXM protocol

\section{3 | RESULTS}

\section{1 | Identification/phenotyping of $\mathbf{T}$ cell and $B$ cell populations}

The cell preparation with the initial negative selection and subsequent separation of mononuclear cells with density gradient centrifugation yielded a highly purified lymphocyte population. The Cellometer software scanned the BR image acquired by the image cytometer and searched for dark membranes of the cells for enumerations. Figure 2A,B show a pure and uniform cell size distribution. The fluorescence optic module VB-595-502 $(525 \pm 32 / 605 \pm 22 \mathrm{~nm})$ collected the emission light from the PE-conjugated anti-human $\operatorname{IgG~F}\left(\mathrm{ab}^{\prime}\right)_{2}$ (Figure 2C) and the fluorescence optic module VB-695-502 $(525 \pm 32 / 695 \pm 30 \mathrm{~nm})$ collected the emission light from the $\mathrm{PE} / \mathrm{Cy} 5$-conjugated anti-CD3 or antiCD19 mAbs to detect $\mathrm{T}$ cells or B cells, respectively (Figure 2D). The fluorescent image segmentations were exported to the FCS Express 4 image cytometry software, where scatter plots were generated using the PE-conjugated anti-IgG $\mathrm{F}\left(\mathrm{ab}^{\prime}\right)_{2}$ and PE/Cy5-conjugated anti-CD3/CD19 fluorescent signals for each sample (Figure 2E).

The percentage of events collected from the scatter plot quadrants 1 and 2 on Figures 3A and 4B representing the T cell population show an average of $71 \%$, while the percentage of events collected from the same quadrants (1 and 2) on
Figures $3 \mathrm{~B}$ and $5 \mathrm{~B}$ representing the $\mathrm{B}$ cell population show an average of $20 \%$. These percentages of $\mathrm{T}$ cells and $\mathrm{B}$ cells are within the range of the normal values demonstrated by flow cytometry on mononuclear cells from healthy subjects. ${ }^{18}$ The overlay analysis of the CD3 and CD19 antigen expression from multiple cells demonstrates that the system is capable of identifying uniform $\mathrm{T}$ cell and $\mathrm{B}$ cell populations as shown in Figure 3C,D, respectively.

\section{2 | Serum titration analysis/linear correlation}

Subsequently, a titration study was performed to evaluate the specificity of the fluorescence signals obtained in the anti-IgG $\mathrm{F}\left(\mathrm{ab}^{\prime}\right)_{2}$-PE channel. A serum sample containing a well-defined DSA profile against the selected target cells was tested at different dilutions (1:1,1:2, and 1:8) (Figures 4 and 5).

Figures 4A and 5A ( $\mathrm{T}$ cells and $\mathrm{B}$ cells, respectively) show that the intensity of the events captured on the digital image by the PE modules (VB-595-502 [525 \pm 32/605 \pm $22 \mathrm{~nm}]$ ) is proportional to the DSA concentration in the sample at different dilutions. The intensity of the events on the PE and PE/Cy5 channels are presented in scatter plots comparable to the flow cytometer analysis (Figures 4B and $5 \mathrm{~B}$ for $\mathrm{T}$ cells and $\mathrm{B}$ cells, respectively). The shift in the anti-IgG $F\left(a b^{\prime}\right)_{2}-\mathrm{PE}$ channel can be also collected in an 
(A)

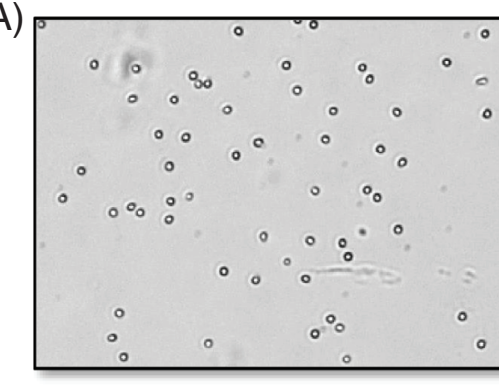

(B)

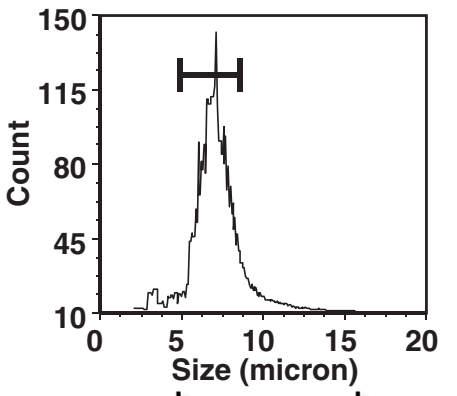

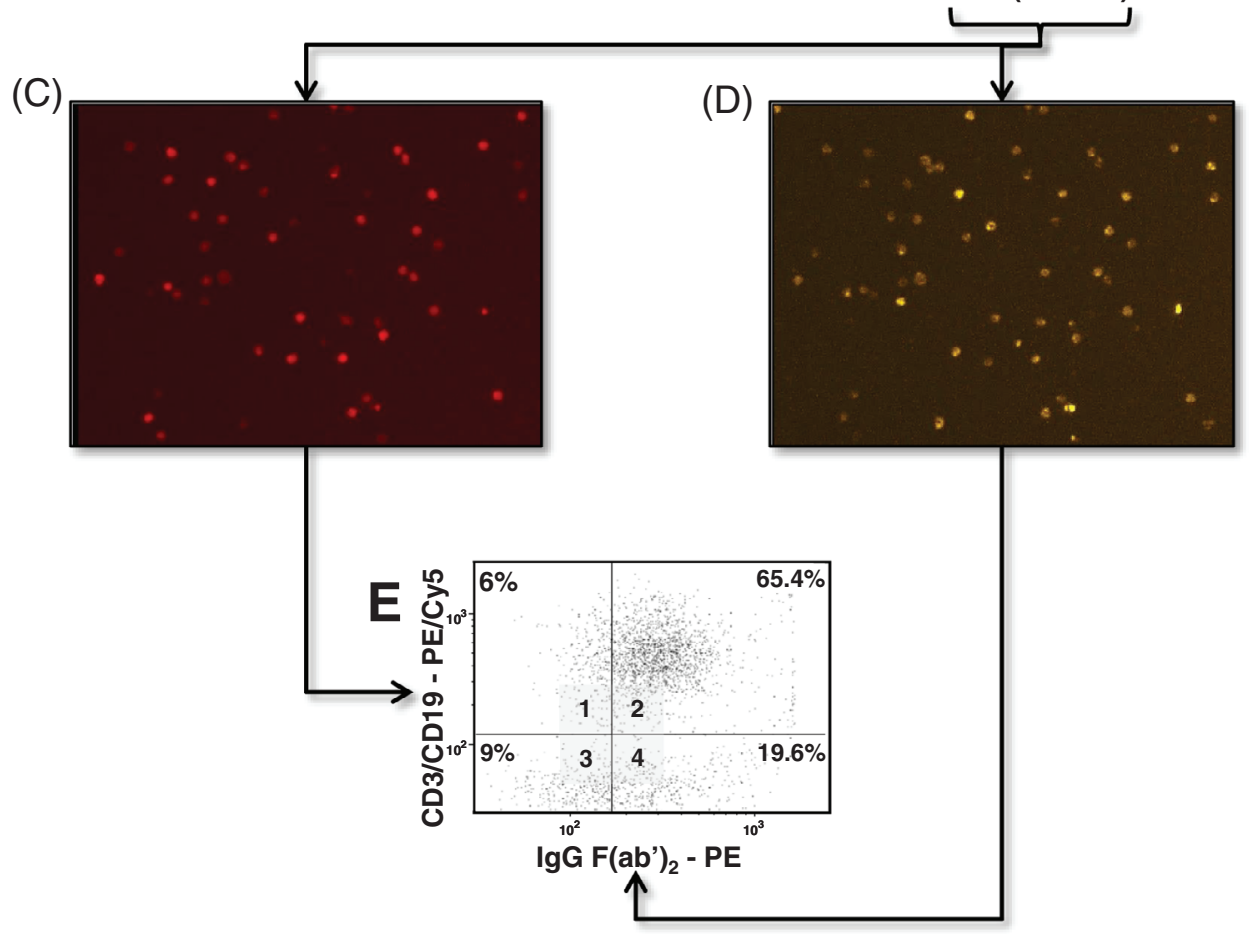

FIGURE 2 Image collection and analysis. Bright field (A), FL1 (C), and FL2 (D) images were from each chamber. The images were segmented and exported to be analyzed by FCS Express 4 image cytometry software. FL1 and FL2 indicate dual-fluorescent

overlay graph for easy comparison with the negative control (Figures 4C and 5C for T cells and B cells, respectively)

Similar serum dilutions/target cell combinations (1:1, $1: 2,1: 4$, and 1:8) were also tested in parallel with the 3 -color FCXM protocol to compare the linearity of the fluorescent signal in both instruments. The fluorescence median value on the PE channel from the Cellometer and the fluorescence median value on the FITC channel from the flow cytometer were transformed into the 256 linear MC for each dilution. The MCS for T cells and B cells crossmatching with each dilution using the FCXM and IXM protocols were analyzed using linear regression. As shown in Figure 6A,B, there was a significant correlation $\left(R^{2}=0.9986\right.$ and 0.9890 , $P<0.01)$ between both systems for $\mathrm{T}$ cell and $\mathrm{B}$ cell crossmatches, respectively.

\section{3 | FCXM vs IXM parallel study}

Using the 3-color FCXM as the reference method, 39 crossmatches were compared with the $\mathrm{T}$ cell and $\mathrm{B}$ cell 2-color IXM. We observed one discrepant result for the T cell crossmatch. The FCXM was positive and the IXM was negative (Table 1), while the formulated VXM was negative in concordance with the IXM due to the absence of detectable DSA against the target cells. On the B cell comparison we observed two discrepant results. Both discrepant results were negative by FCXM and positive by IXM (Table 1). One sample showed DSA against HLA-DR13 (MFI: 5386), and the other sample showed DSA against HLA-Cw7 (MFI: 5386) and HLA-DQ7 (MFI: 5899). Of note, in this last sample the T cell crossmatch results for this serum/cell combination were positive with both the FCXM and the IXM protocols. The predicted positive VXM for both samples were in concordance with the IXM due to the presence of detectable DSA by SAB screening methods. Table 1 summarizes the results of the parallel study, with an overall concordance of $96 \%$, and highly significant sensitivity $(94.1 \%$ and $100 \%)$ and specificity $(100 \%$ and $88.9 \%)$ for T cells and B cells, respectively $(P<0.0001)$.

\section{4 | DISCUSSION}

In this study, we investigated whether the crossmatch assay can be analyzed and interpreted by the Cellometer Vision 
(A)

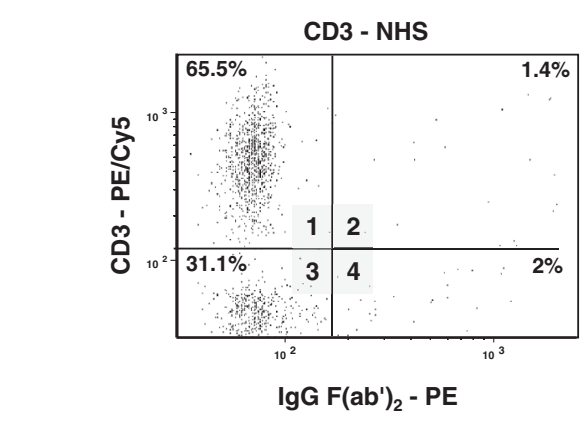

(C)

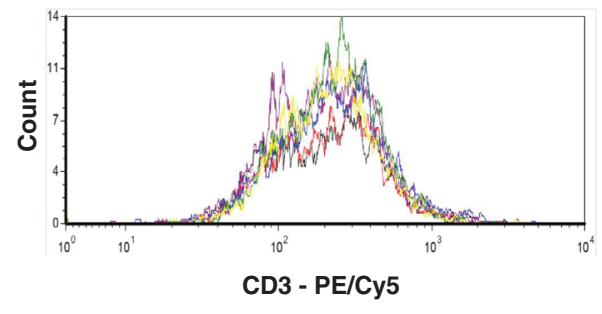

(B)

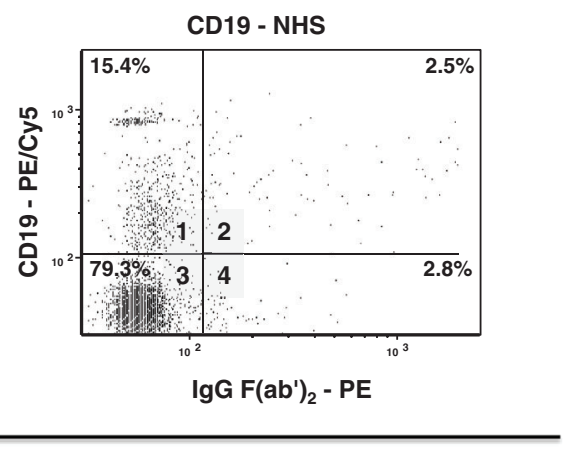

(D)

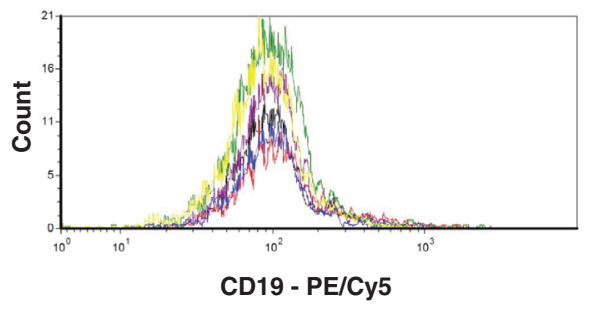

FIGURE 3 Uniform T cell (CD3+) and B cell (CD19+) populations (PE/Cy5). T cell (A) and B cell (B) percentages within the range of normal values. The overlay analysis of the CD3 and CD19 antigen expression from multiple cells demonstrate that the system is capable of identifying uniform T cell (C) and B cell (D) populations, respectively. NHS, normal human serum

(A)

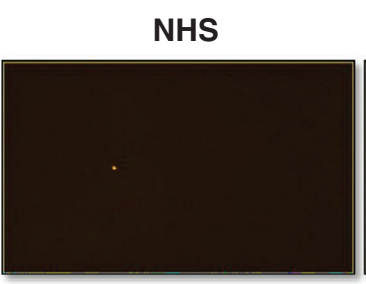

$1: 8$

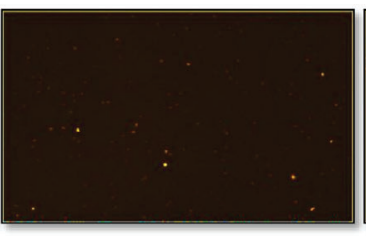

$1: 2$

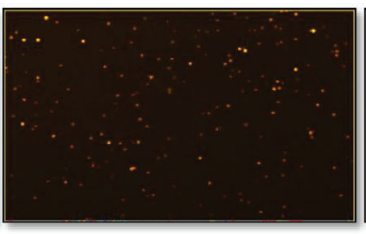

$1: 1$

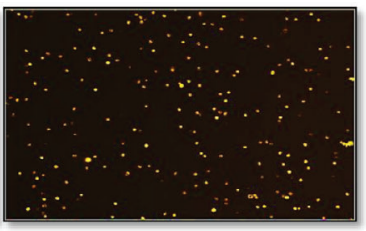

(B)
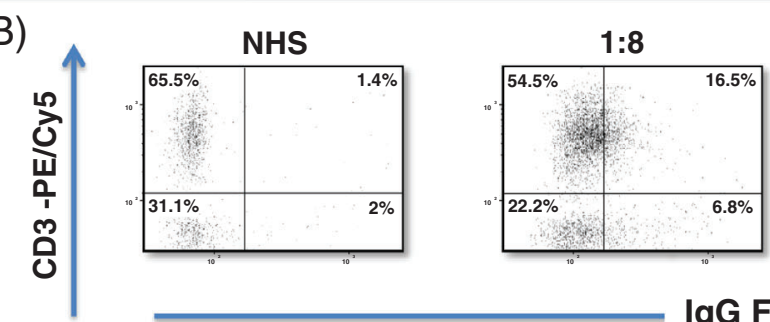

$1: 2$

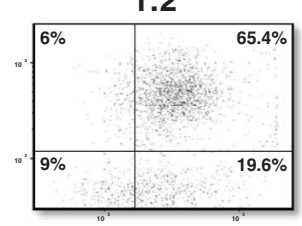

$1: 1$

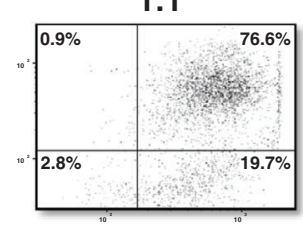

$\lg \mathrm{F}\left(\mathrm{ab}^{\prime}\right)_{2}-\mathrm{PE}$

(C)

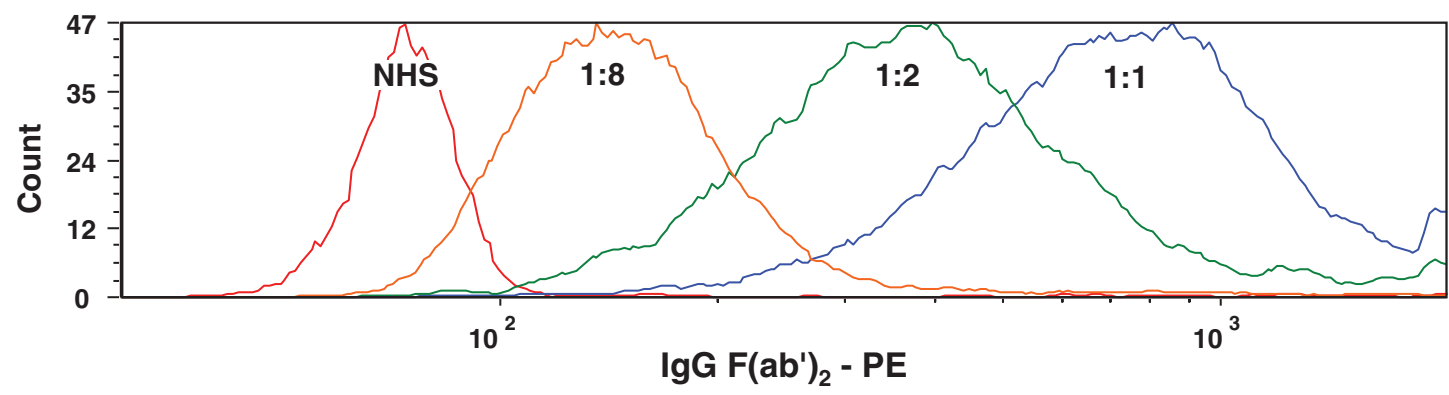

FIGURE 4 T cell image cytometry crossmatch (IXM) serum titration analysis. A, Intensity of the events captured on digital image by PE modules at different dilutions. B, Comparable to flow cytometer analysis, the intensity of the events is presented in scatter plots. C, The cell population shift is represented in an overlay graph for easy comparison with the negative control (NHS). NHS, normal human serum 
(A)
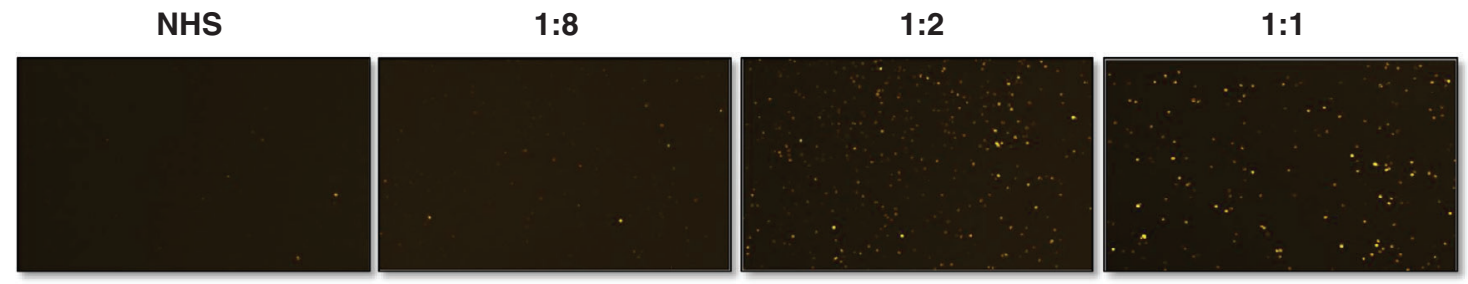

(B)
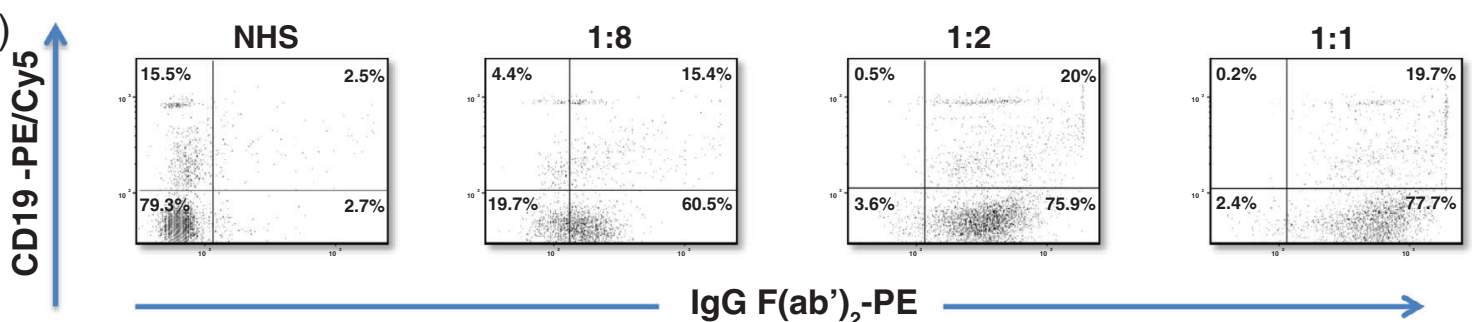

(C)

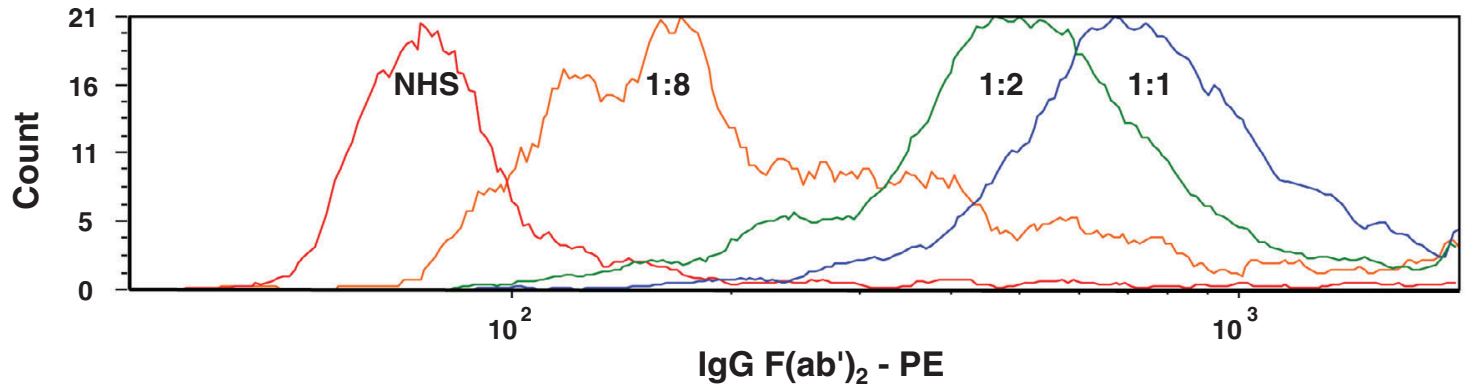

FIGURE 5 B cell image cytometry crossmatch (IXM) serum titration analysis. A, Intensity of the events captured on digital image by PE modules at different dilutions. B, Comparable to flow cytometer analysis, the intensity of the events is presented in scatter plots. C, The cell population shift is represented in an overlay graph for easy comparison with the negative control (NHS). NHS, normal human serum

(A)

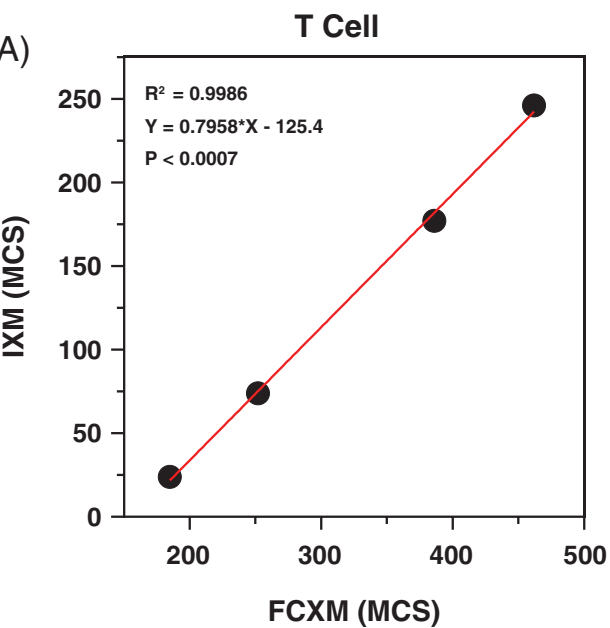

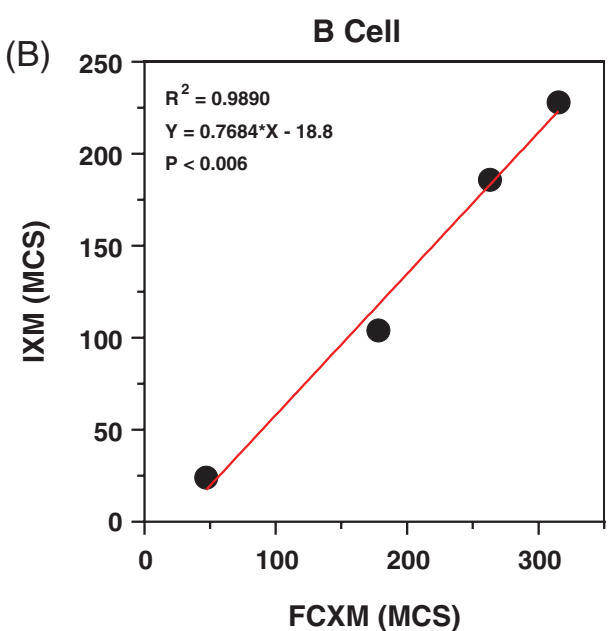

FIGURE 6 Correlation between image cytometry crossmatch (IXM) and flow cytometry crossmatch (FCXM). The T cell (A) and B cell (B) FCXM were performed in parallel using the IXM 2-color protocol and analyzed with the image cytometer, and the 3-color protocol was analyzed with the flow cytometer. Median channel shift (MCS) from both systems were analyzed by linear regression, showing a significant correlation

CBA image cytometer. The results from the IXM show a 96\% overall concordance when compared with the reference FCXM method. The results from this proof of concept study demonstrate that the adapted 2-color IXM protocol from our 3-color FCXM protocol could be successfully analyzed and interpreted using the image cytometer.
The results generated from the fluorescent images captured by the image cytometer and the subsequent analysis of these images, using the FCS Express 4 image cytometry software, allowed the correct characterization of the $\mathrm{T}$ cell and $\mathrm{B}$ cell populations at the expected proportions of healthy subjects. The correct identification of the target $\mathrm{T}$ cells or $\mathrm{B}$ cells 
TABLE 1 Performance characteristics of IXM compared to FCXM

\begin{tabular}{|c|c|c|c|c|}
\hline & \multicolumn{2}{|c|}{ T cell $(n=39)$} & \multicolumn{2}{|c|}{ B cell $(n=39)$} \\
\hline & \multicolumn{2}{|l|}{ IXM } & \multicolumn{2}{|l|}{ IXM } \\
\hline & Positive & Negative & Positive & Negative \\
\hline \multicolumn{5}{|l|}{ FCXM } \\
\hline Positive & 16 & 1 & 21 & 0 \\
\hline Negative & 0 & 22 & 2 & 16 \\
\hline Sensitivity (95\% CI) & \multicolumn{2}{|c|}{$94.1 \%(71.3 \%-99.9 \%)$} & \multicolumn{2}{|c|}{$100 \%(83.9 \%-100 \%)$} \\
\hline Specificity (95\% CI) & \multicolumn{2}{|c|}{$100 \%(84.6 \%-100 \%)$} & \multicolumn{2}{|c|}{$88.9 \%(65.3 \%-98.6 \%)$} \\
\hline PPV (95\% CI) & \multicolumn{2}{|c|}{$100 \%(79.4 \%-100 \%)$} & \multicolumn{2}{|c|}{$91.3 \%(72.0 \%-98.9 \%)$} \\
\hline NPV (95\% CI) & \multicolumn{2}{|c|}{$95.7 \%(78.1 \%-99.9 \%)$} & \multicolumn{2}{|c|}{$100 \%(79.4 \%-100 \%)$} \\
\hline$P$-value & \multicolumn{2}{|l|}{$<0.001$} & \multicolumn{2}{|l|}{$<0.001$} \\
\hline
\end{tabular}

Abbreviations: CI, confidence interval, FCXM, flow cytometry crossmatch; IXM, image cytometry crossmatch; NPV, negative predictive value; PPV, positive predictive value.

is the first step in specificity identification of anti-HLA class I or class II antibodies; later this information will be correlated with the detection of DSA on the SAB solid-phase assay.

The titration experiment with a sample containing known DSA against both HLA class I and class II molecules proves that the system is capable of detecting different concentrations of DSA reacting with T cells and B cells. An excellent linear correlation was obtained when the IXM fluorescent values for each dilution were compared with the results obtained with the FCXM (Figure 6). These results demonstrate that the linearity and sensitivity are comparable for both instruments.

The parallel study of 39 crossmatches by IXM using the FCXM as the reference method showed excellent sensitivity and specificity (Table 1). Three discrepant results were observed, one for $\mathrm{T}$ cell crossmatch and two for $\mathrm{B}$ cell crossmatches. In all these cases, the IXM showed a better correlation with VXM due to the absence or presence of DSA on the SAB assay.

The main limitation of our study is the small number of tested samples; a proper validation will require a larger number of serum and cells samples to adequately survey the extensive polymorphism and expression variation of the HLA system and in the anti-HLA antibodies present in the serum of sensitized patients. Additionally, the protocol will need to be performed in multiple laboratories to demonstrate the robustness and reproducibility of this protocol. Another limitation of this study is the lack of correlation with transplant outcome; however, the clinical utility of optical alloantibody detection using immunofluorescent techniques was previously described. ${ }^{19}$ Using an indirect immunofluorescence crossmatch examined by a phase-contrast fluorescence microscopy, Lobo et $\mathrm{al}^{19}$ showed a significantly higher sensitivity than the CDCXM and a better clinical correlation with accelerated rejection. These results, together with those obtained here in the parallel study with the FCXM, allow us to estimate that the IXM would have a comparable clinical utility to the FCXM, thus giving it the potential to be used in the clinical setting for organ distribution, donor selection, and post-transplant risk assessment.
The utilization of the image cytometry method offers the advantages of automated and objective data collection for reliable analyses of the crossmatch samples without possible variation by different operators. In addition, a larger number of cells are surveyed, and the data is immediately analyzed by the FCS Express 4 image cytometry software.

In addition to the concordance between the IXM and the FCXM results, the image cytometry method has several technical advantages over conventional flow cytometry. One major improvement was the ability to analyze CD3/CD19 expression and $\operatorname{IgG}$ binding simultaneously on the $\mathrm{T}$ cell and $\mathrm{B}$ cell populations, generating the same data plot with the FCS Express 4 image cytometry software as flow cytometry. Using PE and PE/Cy5 fluorescent labels without emission spectral overlapping, fluorescent images for both channels were generated without optical crosstalk and filter set optimization. Furthermore, the lack of high-power lasers or photo-multiplying tubes in the image cytometer eliminates the need for precise optical alignment, where the simple epifluorescence setup does not require daily user maintenance.

Finally, because of the simplicity of the optical architecture, the Cellometer Vision CBA image cytometer does not have a complex fluidic system to calibrate or maintain. Bench-top flow cytometers can be cost efficient, but still have the chance of clogging, risking the sample integrity or significantly delaying the results if no backup is available. Because of the absence of a fluidic system to prime, there is less sample requirement. While most of the FCXM protocols need 200 to $500 \mu \mathrm{L}$, only $20 \mu \mathrm{L}$ of sample is required using the image cytometry method. The acquisition of a flow cytometer is the insurmountable limitation for many laboratories with limited budget. Typically, a standard flow cytometer requires an investment over US\$100 000, where annual maintenance fees cost approximately $10 \%$ of the instrument. This new generation of image cytometer instruments typically costs less than US\$30 000 with no required maintenance fees.

In conclusion, the results presented herein validated the capability and reliability of the Cellometer Vision CBA image cytometer as a dependable alternative instrument for the crossmatch assay. The easy operation without the additional cost and effort of the daily calibration, in addition to the low acquisition cost, make this instrument an excellent alternative for histocompatibility laboratories.

\section{CONFLICTS OF INTEREST}

D.S.R., A.J., and T.F. declare no conflict of interest. L.L.C. and B.D.P. declare competing financial interests, who are employees of Nexcelom Bioscience LLC. The research in this manuscript utilized the Cellometer Vision, which is a product of Nexcelom Bioscience LLC, for developing the cross matching detection method. L.L.C. and B.D.P. provided method development and data analysis for results collected in each experiment. 


\section{ORCID}

Daniel S. Ramon (D) https://orcid.org/0000-0002-3067-5653

\section{REFERENCES}

1. Patel R, Terasaki PI. Significance of the positive crossmatch test in kidney transplantation. N Engl J Med. 1969;280(14):735-739. https://doi.org/10. 1056/NEJM196904032801401.

2. Johnson AH, Rossen RD, Butler WT. Detection of alloantibodies using a sensitive antiglobulin microcytotoxicity test - identification of low-levels of preformed antibodies in accelerated allograft rejection. Tissue Antigens. 1972;2(3):215-216

3. Amos DB, Bashir H, Boyle W, Macqueen M, Tiilikai A. A simple micro cytotoxicity test. Transplantation. 1969;7(3):220-223. https://doi.org/10. 1097/00007890-196903000-00023.

4. Garovoy MR, Rheinschmidt MA, Bigos M, et al. Flow cytometry analysis: a high technology crossmatch technique facilitating transplantation. Transplant Proc. 1983;15(3):1939-1944.

5. Bray RA. Flow cytometry crossmatching for solid organ transplantation. Methods Cell Biol. 1994;41:103-119.

6. Bray RA, Lebeck LK, Gebel HM. The flow cytometric crossmatch. Dual-color analysis of T cell and B cell reactivities. Transplantation. 1989;48(5):834-840.

7. Scornik JC, Bray RA, Pollack MS, et al. Multicenter evaluation of the flow cytometry T-cell crossmatch: results from the American Society of Histocompatibility and Immunogenetics-College of American pathologists proficiency testing program. Transplantation. 1997;63(10):1440-1445.

8. Jaramillo A, Ramon DS, Stoll ST. Technical aspects of crossmatching in transplantation. Clin Lab Med. 2018;38(4):579-593. https://doi.org/10.1016/ j.cll.2018.07.002.

9. Lazda VA, Pollak R, Mozes MF, Jonasson O. The relationship between flow cytometer crossmatch results and subsequent rejection episodes in cadaver renal allograft recipients. Transplantation. 1988;45(3):562-565.

10. Mahoney RJ, Ault KA, Given SR, et al. The flow cytometric crossmatch and early renal transplant loss. Transplantation. 1990;49(3):527-535.

11. Talbot D, Givan AL, Shenton BK, Stratton A, Proud G, Taylor RM. The relevance of a more sensitive crossmatch assay to renal transplantation. Transplantation. 1989;47(3):552-555.

12. Karpinski M, Rush D, Jeffery J, et al. Flow cytometric crossmatching in primary renal transplant recipients with a negative anti-human globulin enhanced cytotoxicity crossmatch. J Am Soc Nephrol. 2001;12(12):28072814.

13. Tambur AR, Ramon DS, Kaufman DB, et al. Perception versus reality?: virtual crossmatch - how to overcome some of the technical and logistic limitations. Am J Transplant. 2009;9(8):1886-1893. https://doi.org/10.1111/j. 1600-6143.2009.02724.x.

14. Cook DJSJ. Flow Cytometry Crossmatch. American Society of Histocompatibility and Immunogenetics Laboratory Manual. 3rd ed. Lenexa, KS: ASHI Press; 1995 .

15. Chan LL, Zhong XM, Pirani A, Lin B. A novel method for kinetic measurements of rare cell proliferation using Cellometer image-based cytometry. J Immunol Methods. 2012;377(1-2):8-14. https://doi.org/10.1016/j.jim.2012. 01.006.

16. Chan LL, Zhong XM, Qiu J, Li PY, Lin B. Cellometer vision as an alternative to flow cytometry for cell cycle analysis, mitochondrial potential, and immunophenotyping. Cytometry Part A. 2011;79A(7):507-517. https://doi. org/10.1002/cyto.a.21071.

17. Chan LLY, Lai N, Wang E, Smith T, Yang X, Lin B. A rapid detection method for apoptosis and necrosis measurement using the Cellometer imaging cytometry. Apoptosis. 2011;16(12):1295-1303. https://doi.org/10.1007/ s10495-011-0651-8.

18. Reichert T, DeBruyere M, Deneys V, et al. Lymphocyte subset reference ranges in adult Caucasians. Clin Immunol Immunopathol. 1991;60(2): 190-208.

19. Lobo PI, Spencer C, Gorman J, Pirsch G. Critical appraisal of complement dependent microlymphocytotoxicity assay for detecting donor-specific alloantibody pretransplant - importance of indirect immunofluorescence as a superior alternative. Hum Immunol. 1981;2(1):55-64.

How to cite this article: Ramon DS, Franks T, Jaramillo A, Paradis BD, Chan LL-Y. Image cytometry as an alternative to flow cytometry for the transplant histocompatibility crossmatch assay. HLA. 2019;93:436-444. https://doi.org/10.1111/tan.13515 